\title{
Optimization of Injectors Location in a Water Reservoirs Aeration Problem
}

\author{
Mohamed Abdelwahed \\ Department of Mathematics, College of Science, King Saud University, Riyadh 11451, Saudi Arabia \\ Correspondence should be addressed to Mohamed Abdelwahed; mabdelwahed@ksu.edu.sa
}

Received 29 September 2012; Revised 29 December 2012; Accepted 13 January 2013

Academic Editor: Ashraf M. Zenkour

Copyright (C) 2013 Mohamed Abdelwahed. This is an open access article distributed under the Creative Commons Attribution License, which permits unrestricted use, distribution, and reproduction in any medium, provided the original work is properly cited.

\begin{abstract}
The aeration process via injectors is used to combat the lack of oxygen in water reservoirs due to the eutrophication problem. The optimization of the injectors location allows to generate a better flow dynamic in order to remedy to this phenomena an preserve the water quality. We propose to adapt the topological gradient method to this problem. The idea consists of studing the topological sensitivity analysis of the considered model based on the three-dimensional-dependent time Navier-Stokes equation with respect to the insertion of an injector in the flow domain. Finally the obtained results are used in a numerical algorithm and some numerical tests are presented to validate the used approach.
\end{abstract}

\section{Introduction}

During the warmer months of the year many lakes and water reservoirs experience some degree of thermal stratification. This phenomena inhibit vertical mixing between the surface layer and the bottom water. This can lead to anoxic conditions in the lower region. The lack of oxygen accelerates the eutrophication of the water which is characterized by a number of damaging effects on its quality. The dynamic aeration is one of used methods to treat the water eutrophication problem. This technique consists of inserting air by the means of injectors located at the bottom of the lake in order to generate a vertical motion mixing up the water of the bottom with that on the top, thus oxygenating the lower part by bringing it in contact with the surface air.

We focus in this work on the optimization of the injectors location in order to generate the best dynamic in the fluid with respect to the aeration purpose. The optimal injectors location is characterized as the solution to a topological optimization problem. The topological sensitivity method is used to solve this problem [1-3]. To present this method, we consider the case in which $\Omega$ contains a single injector $\omega_{z, \mathcal{\varepsilon}}$ located at $z \in \mathbb{R}^{3}$ and having a diameter $\varepsilon$. The topological sensitivity method provides an asymptotic expansion of an objective function $j$ of the form

$$
j\left(\Omega \backslash \overline{\omega_{z, \varepsilon}}\right)=j(\Omega)+\varepsilon \delta j(z)+o(\varepsilon), \quad \forall z \in \Omega .
$$

This expression is called the topological asymptotic expansion and $\delta j$ is the topological sensitivity function. In order to minimize the cost function, the best location to insert the injector in $\Omega$ is where $\delta j$ is the most negative. In fact if $\delta j(z)<$ 0 , we have $j\left(\Omega \backslash \overline{\omega_{z \varepsilon}}\right)<j(\Omega)$ for small $\varepsilon$. The function $\delta j$ can be used as a descent direction in the domain optimization process.

This method is used in [4] to optimize the injector position. In this work a stationary model based on generalized Stokes equations is considered. This paper extend this approach to dependent time model based on three dimensional Navier-Stokes equations. In order to obtain a topological sensitivity analysis of the used model with respect to the insertion of an injector, the model is firstly discretized on time using the characteristics method. A generalized Stokes problem is obtained at each time step. The topological sensitivity analysis for this problem presented in [4] is used and then extended to the Navier-Stokes problem.

The paper is organized as follows. The used model is presented in Section 2. Section 3 is devoted to the optimization 
problem. A topological sensitivity analysis for the generalized Stokes equations is presented and extended to Navier-Stokes equations. Finally, we illustrate the efficiency of the proposed method by several numerical experiments in Section 4 .

\section{Physical Model}

Let $\Omega$ be a three-dimensional flow domain representing the eutrophized lake. Different models can be used to describe the resulting two phase water air bubbles flow problem. A general framework is proposed in [5]. In our case, such a problem leads to many difficulties mainly due to the complexity of the model itself and to the inherent high computing costs for such large-scale applications. For this reason, Legendre in [6] studied the case of one air bubble and its interactions with the fluid flow. This work has been extended to several air bubbles by Clement in [7] but in two-dimensional case. Using the fact that the water phase is dominant, a two-dimensional simplified model is proposed in [8]. In this work, the water phase is governed by the three-dimensional dependent time Navier-Stokes equations in which we integrate the effect of momentum released by the injected bubbles by adding a local boundary condition for the velocity on the injector holes. In the presence of an injector $\omega_{\text {inj }} \subset \Omega$ (see Figure 1 ), the velocity $u(x, t)$ and the pressure $p(x, t)$ solve the following system:

$$
\begin{aligned}
& \left.\left.\frac{\partial u}{\partial t}+u \cdot \nabla u-v \Delta u+\nabla p=\mathscr{G} \quad \text { in } \Omega_{i} \times\right] 0, T\right] \\
& \left.\left.\nabla \cdot u=0 \quad \text { in } \quad \Omega_{i} \times\right] 0, T\right] \\
& u(\cdot, 0)=u^{0} \text { in } \Omega_{i}, \\
& \left.\left.\sigma(u, p) \cdot n=0 \quad \text { on } \Gamma_{n} \times\right] 0, T\right], \\
& \left.\left.u=u_{\text {wind }} \quad \text { on } \Gamma_{s} \times\right] 0, T\right] \\
& \left.\left.u=0 \quad \text { on } \Gamma_{w} \times\right] 0, T\right] \\
& \left.\left.u=u_{\text {inj }} \quad \text { on } \partial \omega_{\text {inj }} \times\right] 0, T\right],
\end{aligned}
$$

where $\Omega_{i}=\Omega \backslash \overline{\omega_{\text {inj }}}$ is the lake domain in the presence of the injector $\omega_{\text {inj }}, v$ is the water viscosity, $\mathscr{G}$ is the gravitational force, $\Gamma_{n}$ is a free boundary (inlet/outlet), $\Gamma_{s}$ is the surface in contact with the atmosphere, $u_{\text {wind }}$ is the wind velocity, $\Gamma_{w}$ is the bottom lake boundary, $T$ is the final time of simulation, $u^{0}$ is the initial velocity field, and $\sigma(u, p)=v\left(\nabla u+\nabla u^{T}\right)-p I$ is stress tensor with $n$ is unit outward normal vector. and by

For the sake of simplicity, we denote by $\Gamma_{i}=\Gamma_{s} \cup \Gamma_{w} \cup \partial \omega_{\text {inj }}$

$$
u_{d}= \begin{cases}u_{\text {wind }} & \text { on } \Gamma_{s} \\ 0 & \text { on } \Gamma_{w} \\ u_{\text {inj }} & \text { on } \partial \omega_{\text {inj }}\end{cases}
$$

\section{Optimization of Injectors Location}

This section is dedicated to design an efficient method to optimize the injectors location in order to generate the best

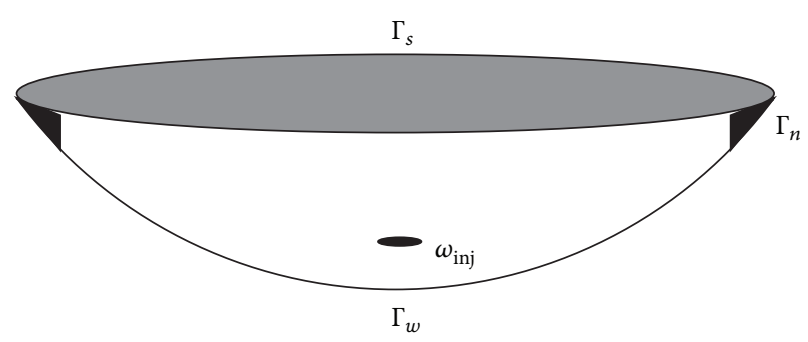

Figure 1: Domain $\Omega$.

motion of the fluid. The topological optimization method is used for this purpose.

3.1. Topological Optimization Problem. For the sake of simplicity, we will assume that the injectors are well separated and have the geometry form $\omega_{z_{k}, \varepsilon}=z_{k}+\varepsilon \omega^{k}, 1 \leq k \leq m$, where $\varepsilon$ is the shared diameter and $\omega^{k} \subset \mathbb{R}^{3}$ are bounded and smooth domains containing the origin. The points $z_{k} \in$ $\Omega, 1 \leq k \leq m$ determine the location of the injectors. The domains $\omega^{k}$ describe the injectors geometries.

In the presence of injectors, the velocity $u_{\varepsilon}$ and the pressure $p_{\varepsilon}$ satisfy the following system:

$$
\begin{gathered}
\frac{\partial u_{\varepsilon}}{\partial t}+\left(u_{\varepsilon} \cdot \nabla\right) u_{\varepsilon}-v \Delta u_{\varepsilon}+\nabla p_{\varepsilon}=\mathscr{G} \\
\text { in } \left.\left.\Omega \backslash \bigcup_{k=1}^{m} \overline{\omega_{z_{k}, \varepsilon}} \times\right] 0, T\right], \\
\left.\left.\nabla \cdot u_{\varepsilon}=0 \quad \text { in } \Omega \backslash \bigcup_{k=1}^{m} \overline{\omega_{z_{k}, \varepsilon}} \times\right] 0, T\right], \\
\left.\left.u_{\varepsilon}=u_{d} \quad \text { on } \Gamma \times\right] 0, T\right], \\
\left.\left.\sigma\left(u_{\varepsilon}, p_{\varepsilon}\right) \cdot n=0 \quad \text { on } \Gamma_{n} \times\right] 0, T\right] .
\end{gathered}
$$

Note that for $\varepsilon=0,\left(u_{0}, p_{0}\right)$ is solution to

$$
\begin{aligned}
& \left.\left.\frac{\partial u_{0}}{\partial t}+\left(u_{0} \cdot \nabla\right) u_{0}-v \Delta u_{0}+\nabla p_{0}=\mathscr{G} \text { in } \Omega \times\right] 0, T\right] \\
& \left.\left.\nabla \cdot u_{0}=0 \quad \text { in } \Omega \times\right] 0, T\right] \\
& \left.u_{0}=u_{d} \quad \text { on }[\times] 0, T\right] \\
& \left.\left.\sigma\left(u_{0}, p_{0}\right) \cdot n=0 \quad \text { on } \Gamma_{n} \times\right] 0, T\right] .
\end{aligned}
$$

For the optimization criteria, we assume that a "good" lake oxygenation can be described by a target velocity $\mathcal{U}_{g}$. Then, the cost function $\mathscr{J}_{\varepsilon}$ to be minimized is given by

$$
\mathscr{J}_{\varepsilon}\left(u_{\varepsilon}\right)=\int_{0}^{T} J_{\varepsilon}\left(u_{\varepsilon}\right) d t \text { with } J_{\varepsilon}\left(u_{\varepsilon}\right)=\int_{\Omega_{m}}\left|u_{\varepsilon}-\mathcal{U}_{g}\right|^{2} d x \text {, }
$$

where $\Omega_{m} \subset \Omega$ is the measurement domain (the top layer, see Figure 2). 


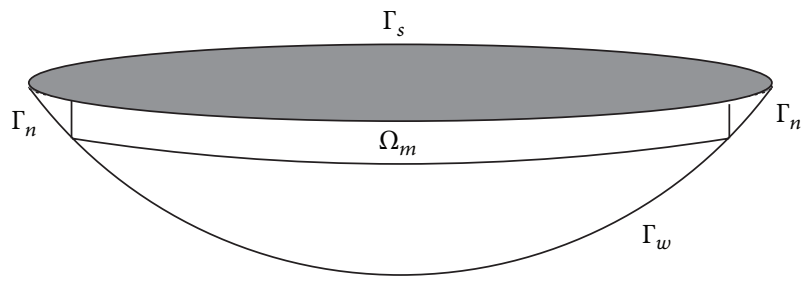

FIgURE 2: The top layer $\Omega_{m}$.

We denote by $j$ the design function defined by

$$
j\left(\Omega \backslash \bigcup_{k=1}^{m} \overline{\omega_{z_{k}, \varepsilon}}\right)=\mathscr{J}_{\varepsilon}\left(u_{\varepsilon}\right),
$$

where $u_{\varepsilon}$ is the solution of (4).

The identification problem can be formulated as a topological optimization problem: find the optimal location of the injectors $\omega_{z_{k}, \varepsilon}=z_{k}+\varepsilon \omega^{k}, 1 \leq k \leq m$, inside the lake domain $\Omega$ minimizing the optimal design function $j$ :

$$
\left(\mathcal{O}_{\varepsilon}\right)\left\{\begin{array}{l}
\text { Find } z_{k}^{*} \in \Omega, 1 \leq k \leq m, \text { such that } \\
j\left(\Omega \backslash \bigcup_{k=1}^{m} \overline{\omega_{z_{k}^{*}, \varepsilon}} \cup_{k=1}^{m}\right)=\min _{\omega_{z_{k}, \varepsilon} \subset \Omega} j\left(\Omega \backslash \bigcup_{k=1}^{m} \overline{\omega_{z_{k}, \varepsilon}}\right) .
\end{array}\right.
$$

To solve this optimization problem $\left(\mathcal{O}_{\varepsilon}\right)$ we will use the topological sensitivity analysis method [2, 3, 9-11]. It consists of studying the variation of the design function $j$ with respect to a small topological perturbation of the domain $\Omega$.

3.2. Time Discretization. The Lagrangian representation of the flow is based on the function $X:(x, s ; t) \in \Omega \times(0, T)^{2} \rightarrow$ $X(x, s ; t)$, where $X(x, s ; t)$ is the position at time $t$ of the particle of fluid which is at point $x$ at time $t=s$. Thus $t \rightarrow X(x, s ; t)$ is the parametric representation of the ajectory of the particle. Given the velocity $u$, the trajectory may be determined from the initial value problem:

$$
\begin{gathered}
\frac{d}{d t} X(x, s ; t)=u(X(x, s ; t), t), \\
X(x, s ; s)=x .
\end{gathered}
$$

Lemma 1. If $|s-t|$ is sufficiently small, then the Jacobian of the homeomorphism $x \rightarrow X(x, s ; t)$ is equal to 1 .

Proof. See [12].

Let NT be a positive integer, $\Delta t=T / \mathrm{NT}$ and $t^{n}=n \Delta t$. For $x \in \Omega, s=t^{n+1}, t^{n} \leq t \leq t^{n+1}, 0 \leq n \leq N$, (9) implies using Euler scheme that

$$
\begin{aligned}
x-X\left(x, t^{n+1} ; t^{n}\right) & =\int_{t^{n}}^{t^{n+1}} u\left(X\left(x, t^{n+1} ; t\right), t\right) d t \\
& \approx \Delta t u\left(X\left(x, t^{n+1} ; t^{n+1}\right), t^{n+1}\right) \\
& =\Delta t u\left(x, t^{n+1}\right) .
\end{aligned}
$$

Then numerically the position $X\left(x, t^{n+1} ; t^{n}\right)$ is determined by

$$
X\left(x, t^{n+1} ; t^{n}\right)=x-\Delta t u\left(x, t^{n+1}\right) .
$$

Using (10) it is shown in [12] that material derivative is as follows:

$$
\frac{d u}{d t}=\frac{\partial u}{\partial t}+(u \cdot \nabla) u=\frac{u\left(x, t^{n+1}\right)-u\left(X\left(x, t^{n+1} ; t^{n}\right), t^{n}\right)}{\Delta t} .
$$

This approximation is called characteristic method [13] and using Lemma 1 we show that (12) is $L^{2}$ stable [14].

Using (12), problem (4) is equivalent to solve the following generalized Stokes equation:

Find $u^{n+1}$ and $p^{n+1}$ solutions of

$$
\begin{aligned}
& \alpha u_{\varepsilon}^{n+1}-v \Delta u_{\varepsilon}^{n+1}+\nabla p_{\varepsilon}^{n+1}=F_{\varepsilon}^{n+1} \quad \text { in } \Omega \backslash \bigcup_{k=1}^{m} \overline{\omega_{z_{k}, \varepsilon}}, \\
& \nabla \cdot u_{\varepsilon}^{n+1}=0 \quad \text { in } \Omega \backslash \bigcup_{k=1}^{m} \overline{\omega_{z_{k},}}, \\
& u_{\varepsilon}^{n+1}=u_{d} \quad \text { on } \Gamma, \\
& \sigma\left(u_{\varepsilon}^{n+1}, p_{\varepsilon}^{n+1}\right) \cdot n=0 \quad \text { on } \Gamma_{n},
\end{aligned}
$$

where $\alpha=1 / \Delta t, F_{\varepsilon}^{n+1}=(1 / \Delta t) u_{\varepsilon}^{n}\left(X\left(x, t^{n+1} ; t^{n}\right)\right)+\mathscr{G}, u_{\varepsilon}^{n+1}$, and $p_{\varepsilon}^{n+1}$ are the approximations of $u_{\varepsilon}$ and $p_{\varepsilon}$ on time $t^{n+1}$. Then, at each time step, we have to solve a steady state generalized Stokes problem having the following generic form:

$$
\begin{gathered}
\alpha u_{\varepsilon}-v \Delta u_{\varepsilon}+\nabla p_{\varepsilon}=F_{\varepsilon} \quad \text { in } \Omega \backslash \bigcup_{k=1}^{m} \overline{\omega_{z_{k}, \varepsilon}}, \\
\nabla \cdot u_{\varepsilon}=0 \quad \text { in } \Omega \backslash \bigcup_{k=1}^{m} \overline{\omega_{z_{k}, \varepsilon}}, \\
u_{\varepsilon}=u_{d} \text { on } \Gamma, \\
\sigma\left(u_{\varepsilon}, p_{\varepsilon}\right) \cdot n=0 \text { on } \Gamma_{n} .
\end{gathered}
$$

Note that for $\varepsilon=0$, at each time step $\left(u_{0}, p_{0}\right)$ is solution to

$$
\begin{gathered}
\alpha u_{0}-v \Delta u_{0}+\nabla p_{0}=F_{0} \quad \text { in } \Omega, \\
\nabla \cdot u_{0}=0 \quad \text { in } \Omega, \\
u_{0}=u_{d} \quad \text { on } \Gamma, \\
\sigma\left(u_{0}, p_{0}\right) \cdot n=0 \quad \text { on } \Gamma_{n} .
\end{gathered}
$$

Then, in order to obtain an asymptotic expansion of the cost function $\mathscr{J}_{\varepsilon}$ for the Navier-Stokes problem, we study first the topological sensitivity analysis for the generalized Stokes problem by giving an asymptotic expansion of the cost function $J_{\varepsilon}$. 
3.3. Topological Sensitivity Analysis for the Generalized Stokes Problem. In this section we present the topological asymptotic expansion of the cost function $J_{\varepsilon}$ defined in (6) with respect to the insertion of a small injector $\omega_{z, \varepsilon}=z+\varepsilon \omega$ inside the domain $\Omega$. From the week formulation of (14) one can show that $u_{\varepsilon}$ is the solution to

$$
\begin{gathered}
u_{\varepsilon} \in \mathscr{V}_{\varepsilon}, \\
\mathscr{A}_{\varepsilon}\left(u_{\varepsilon}, w\right)=b_{\varepsilon}(w), \quad \forall w \in \mathscr{V}_{\varepsilon}^{0}, \\
u_{\varepsilon_{\mid \Gamma}}=u_{d}, \quad u_{\varepsilon_{\mid \partial \omega_{z, \varepsilon}}}=u_{\mathrm{inj}},
\end{gathered}
$$

where the functional space $\mathscr{V}_{\varepsilon}$, the bilinear form $\mathscr{A}_{\varepsilon}$, and the linear form $b_{\varepsilon}$ are defined by

$$
\begin{gathered}
\mathscr{V}_{\varepsilon}=\left\{\theta \in H^{1}\left(\Omega \backslash \overline{\omega_{z, \varepsilon}}\right)^{3}, \operatorname{div} \theta=0 \text { in } \Omega \backslash \overline{\omega_{z, \varepsilon}}\right\}, \\
\mathscr{V}_{\varepsilon}^{0}=\mathscr{V}_{\varepsilon} \cap H_{0}^{1}\left(\Omega \backslash \overline{\omega_{z, \varepsilon}}\right)^{3}, \\
\mathscr{A}_{\varepsilon}(u, w)=\alpha \int_{\Omega \backslash \overline{\omega_{z, \varepsilon}}} u w d x+v \int_{\Omega \backslash \overline{\omega_{z, \varepsilon}}} \nabla u \nabla w d x \\
\forall u, w \in \mathscr{V}_{\varepsilon}, \\
b_{\varepsilon}(w)=\int_{\Omega \backslash \overline{\omega_{z, \varepsilon}}} F_{\varepsilon} w d x \quad \forall w \in \mathscr{V}_{\varepsilon} .
\end{gathered}
$$

Next, we denote by $\widehat{u}_{\varepsilon}$ the following extension of $u_{\varepsilon}$ in $\Omega$ :

$$
\widehat{u}_{\varepsilon}= \begin{cases}u_{\varepsilon} & \text { in } \Omega, \\ u_{\text {inj }} & \text { in } \omega_{z, \varepsilon} .\end{cases}
$$

We begin first by presenting the topological asymptotic expansion of a cost function $J_{\varepsilon}$ defined on $\mathscr{V}_{\varepsilon}$ and verifying the following hypothesis.

Hypothesis 1. (i) $J_{0}$ is differentiable with respect to $u$; its derivative is denoted by $D J_{0}(u)$.

(ii) There exists a real number $\delta J$ such that $\forall \varepsilon \geq 0$

$$
J_{\varepsilon}\left(u_{\varepsilon}\right)-J_{0}\left(u_{0}\right)=D J_{0}\left(u_{0}\right)\left(\widehat{u}_{\varepsilon}-u_{0}\right)+\varepsilon \delta J+o(\varepsilon) .
$$

(iii) The adjoint problem

$$
\mathscr{A}_{0}\left(v_{0}, w\right)=-D J_{0}\left(u_{0}\right) w, \quad \forall w \in \mathscr{V}_{0}^{0},
$$

has a unique solution $v_{0} \in \mathscr{V}_{0}^{0}$.

The asymptotic expansion described in the following theorem is valid for arbitrary shaped holes and all cost function verifying the Hypothesis 1 . The case of spherical holes is given in (24) [11]. Finally we present the sensitivity analysis corresponding to the cost function $J_{\varepsilon}$ defined in (6).

Theorem 2. If Hypothesis 1 holds, the function $J_{\varepsilon}$ has the following asymptotic expansion:

$$
\begin{aligned}
J_{\varepsilon} & \left(u_{\varepsilon}\right)-J_{0}\left(u_{0}\right) \\
& =\varepsilon\left[\left(-\int_{\partial \omega} \eta(y) d s(y)\right) \cdot v_{0}(z)+\delta J\right]+\Delta t o(\varepsilon),
\end{aligned}
$$

where $\eta \in H^{-1 / 2}(\partial \omega)^{3}$ is the solution to the boundary integral equation

$$
\int_{\partial \omega} E(y-x) \eta(x) d s(x)=u_{i n j}-u_{0}(z), \quad \forall y \in \partial \omega,
$$

with $(E, \Pi)$ is the fundamental solution of the Stokes equations: $E(y)=(1 / 8 \pi v r)\left(I+e_{r} e_{r}^{T}\right), \Pi(y)=y / 4 \pi r^{3}$ with $r=\|y\|$, $e_{r}=y / r$ and $e_{r}^{T}$ is the transposed vector of $e_{r}$.

Proof. This proof is an adaptation of the proof presented in [11].

In the particular case where $\omega=B(0,1)$, the density $\eta$ is given explicitly by [10]

$$
\eta(y)=-\frac{3 v}{2}\left(u_{0}(z)-u_{\text {inj }}\right), \quad \forall y \in \partial \omega .
$$

In this case and under the hypothesis of Theorem 2, the asymptotic expansion becomes

$$
\begin{aligned}
& J_{\varepsilon}\left(u_{\varepsilon}\right)-J_{0}\left(u_{0}\right) \\
& \quad=\varepsilon\left[6 \pi v\left(u_{0}(z)-u_{\mathrm{inj}}\right) \cdot v_{0}(z)+\delta J\right]+\Delta t o(\varepsilon) .
\end{aligned}
$$

Proposition 3. The function $J_{\varepsilon}$ given by (6) satisfies the Hypothesis 1 with

$$
D J_{0}\left(u_{0}\right)(w)=2 \int_{\Omega_{m}}\left(u_{0}-\mathscr{U}_{g}\right) w d x, \quad \forall w \in \mathscr{V}_{0}, \delta J=0 .
$$

Then, The function $J_{\varepsilon}$ has the following expansion

$$
J_{\varepsilon}\left(u_{\varepsilon}\right)-J_{0}\left(u_{0}\right)=2 \int_{\Omega_{m}}\left(u_{0}-\mathscr{U}_{g}\right)\left(\widehat{u}_{\varepsilon}-u_{0}\right) d x+\Delta t o(\varepsilon) \text {. }
$$

Proof. See [15].

3.4. Topological Sensitivity Analysis for the Navier-Stokes Problem. In this section we consider the in stationary NavierStokes problem (4). We want to compute the variation $j(\Omega \backslash$ $\left.\overline{\omega_{z, \varepsilon}}\right)-j(\Omega)$ with respect to the insertion of a small injector in the fluid flow domain. by

Using (6), the variation of the design function (7) is given

$$
\begin{aligned}
j\left(\Omega \backslash \overline{\omega_{z, \varepsilon}}\right)-j(\Omega) & =\mathscr{J}_{\varepsilon}\left(u_{\varepsilon}\right)-\mathscr{J}_{0}\left(u_{0}\right) \\
& =\int_{0}^{T} J_{\varepsilon}\left(u_{\varepsilon}\right) d t-\int_{0}^{T} J_{0}\left(u_{0}\right) d t \\
& =\sum_{n=1}^{\mathrm{NT}} \int_{t^{n-1}}^{t^{n}}\left(J_{\varepsilon}\left(u_{\varepsilon}\right)-J_{0}\left(u_{0}\right)\right) d t,
\end{aligned}
$$

where NT $\in \mathbb{N}^{*}$ and $t^{n}=n \Delta t$, with $\Delta t=T / \mathrm{NT}$ is the time step. 
By trapezoidal formula, we get

$$
\begin{aligned}
& \int_{t^{n-1}}^{t^{n}}\left(J_{\varepsilon}\left(u_{\varepsilon}(\cdot, t)\right)-J_{0}\left(u_{0}(\cdot, t)\right)\right) d t \\
& \quad=\frac{\Delta t}{2}\left[\left(J_{\varepsilon}\left(u_{\varepsilon}^{n}\right)-J_{0}\left(u_{0}^{n}\right)\right)+\left(J_{\varepsilon}\left(u_{\varepsilon}^{n-1}\right)-J_{0}\left(u_{0}^{n-1}\right)\right)\right] .
\end{aligned}
$$

Using the sensitivity analysis for the generalized Stokes equations (21) and Proposition 3, we deduce the following asymptotic expansion

$$
j\left(\Omega \backslash \overline{\omega_{z, \varepsilon}}\right)-j(\Omega)=\frac{1}{2} \varepsilon \Delta \operatorname{tg}(z)+o(\varepsilon),
$$

where $g(z)=\sum_{n=1}^{\mathrm{NT}}\left(\delta j^{n}(z)+\delta j^{n-1}(z)\right)$, with

$$
\delta j^{n}(z)=\left(-\int_{\partial \mathcal{O}} \eta^{n}(y) d s(y)\right) \cdot v_{0}^{n}(z) .
$$

Here $v_{0}^{n}$ and $\eta^{n}$ are, respectively, the approximations at time $t^{n}=n \Delta t$ of the adjoint solution $v_{0}(\cdot t)$ of problem $(20)$ and $\eta$ defined in (22). If $\omega$ is the unit ball $B(0,1)$, by (24)

$$
\delta j^{n}(z)=6 \pi v\left(u_{0}^{n}(z)-u_{\text {inj }}\right) \cdot v_{0}^{n}(z) .
$$

In this case, using (25). The design function $j$ defined in (7) has the following expansion:

$$
\begin{aligned}
& j\left(\Omega \backslash \overline{\omega_{z, \varepsilon}}\right)-j(\Omega) \\
& =3 \pi v \varepsilon \Delta t \\
& \quad \times \sum_{n=1}^{\mathrm{NT}}\left[\left(u_{0}^{n}(z)-u_{\mathrm{inj}}\right) \cdot v_{0}^{n}(z)\right. \\
& \left.\quad+\left(u_{0}^{n-1}(z)-u_{\mathrm{inj}}\right) \cdot v_{0}^{n-1}(z)\right]+o(\varepsilon),
\end{aligned}
$$

where $u_{0}^{n}$ and $v_{0}^{n}$ are, respectively, solutions to the generalized Stokes problem (15) and its associated adjoint problem (20).

\section{Numerical Results}

This section is devoted to some numerical examples. Based on the previous theoretical results, we propose fast and accurate algorithms for the detection of optimal injectors location.

As described in the previous section, each injector $\operatorname{Inj}_{k}, 1 \leq k \leq m$, is modeled as a small hole $\omega_{z_{k}, \varepsilon}=z_{k}+\varepsilon \omega^{k}$ around $z_{k} \in \Omega$, having an injection velocity $u_{\text {inj. }}^{k}$. In order to use the explicit topological sensitivity expression we assume that $\omega^{k}=B(0,1)$.

We recall that the optimal injectors location is characterized as the solution of the topological optimization problem

$$
\min _{\omega_{z_{k}, \varepsilon} \subset \Omega} \int_{0}^{T}\left(\int_{\Omega_{m}}\left|u_{\varepsilon}-\mathcal{U}_{g}\right|^{2} d x\right) d t,
$$

where $\Omega_{m}$ is a measurement domain (see Figure 2), $\mathcal{U}_{g}$ is an objective fluid flow, and $u_{\varepsilon}$ is the solution to the Navier-Stokes equations in $\Omega \backslash \cup_{k=1}^{m} \overline{\omega_{z_{k}, \varepsilon}}$. rithm.
Algorithm 4. (i) For $n=1, \mathrm{NT}$

(a) compute $u_{0}^{n}$, a solution to the generalized Stokes equations (15),

(b) compute $v_{0}^{n}$, a solution to the associated adjoint problem (20).

(ii) For $1 \leq k \leq 3$, compute the sensitivity

$$
\begin{aligned}
& \delta j^{k}(z)=\sum_{n=1}^{\mathrm{NT}}\left[\left(u_{0}^{n}(z)-u_{\mathrm{inj}}^{k}\right) \cdot v_{0}^{n}(z)\right. \\
&\left.+\left(u_{0}^{n-1}(z)-u_{\text {inj }}^{k}\right) \cdot v_{0}^{n-1}(z)\right], \\
& \forall z \in \Omega .
\end{aligned}
$$

We note that the topological sensitivity function $\delta j^{k}$ depends on $u_{0}^{n}$ and $v_{0}^{n}$ solutions, respectively, to the direct and adjoint problems computed in the domain $\Omega$ (not in the perturbed one $\Omega \backslash \overline{\omega_{z, \varepsilon}}$ ) which is an interesting feature of the used approach. The mixed finite element method " $P^{1}+$ bubble $/ P^{1 "}[16]$ is used for the space approximation of the problems (15) and (20). The obtained linear system is solved using Uzawa method [13]. One can consult [12] for the existence, uniqueness of the solution and the analysis of the error estimates of the discrete solution $\left(u_{h}, p_{h}\right)$.

In numerical tests, different values for NT and $\Delta t$ are studied. The presented results in Figures 3-9 correspond to $\mathrm{NT}=60$ and $\Delta t=1 s$.

Next, some numerical examples are presented. The first and second tests presented in Sections 4.1 and 4.2 concern, respectively, the study of the injector radius and the mesh step $h$ effects on the optimization precess. We consider $\Omega=[0,1] \times$ $[0,1] \times[0,1]$. The domain $\Omega$ is discretized by tetrahedral mesh with mesh step $h$.

The third example concerns the identification of separated small injectors in a water reservoir. In the last example we discuss the detection of an injector line containing a sequence of small injectors representing the injection holes. For the two last tests presented in Sections 4.3 and 4.4, a 3D reservoir with length $8 \mathrm{~m}$, width $4 \mathrm{~m}$, and maximum height $3 \mathrm{~m}$ is considered. The domain is discretized using 16007 nodes and 85112 tetrahedral.

4.1. Sensitivity to the Injector's Radius. The goal is to study the injector (considered as a sphere) radius effect $r$. We consider the case of a unique injector $\omega$ centered at $z^{*}$ and having radius $r$. We introduce the following error function:

$$
\operatorname{er}(h, r)=\frac{\left\|z_{h}-z^{*}\right\|}{2 r},
$$

where $\|\cdot\|$ is the usual norm in $\mathbb{R}^{3}, z^{*}$ is the exact injector location, and $z_{h}$ is the computed location using the mesh step $h$. We presenet in Figure 3 the obtained errors for $h=0.02$ and different injector radius $r$ varying from 0.02 to 0.1 . We remark that error rises when $r$ increases. 


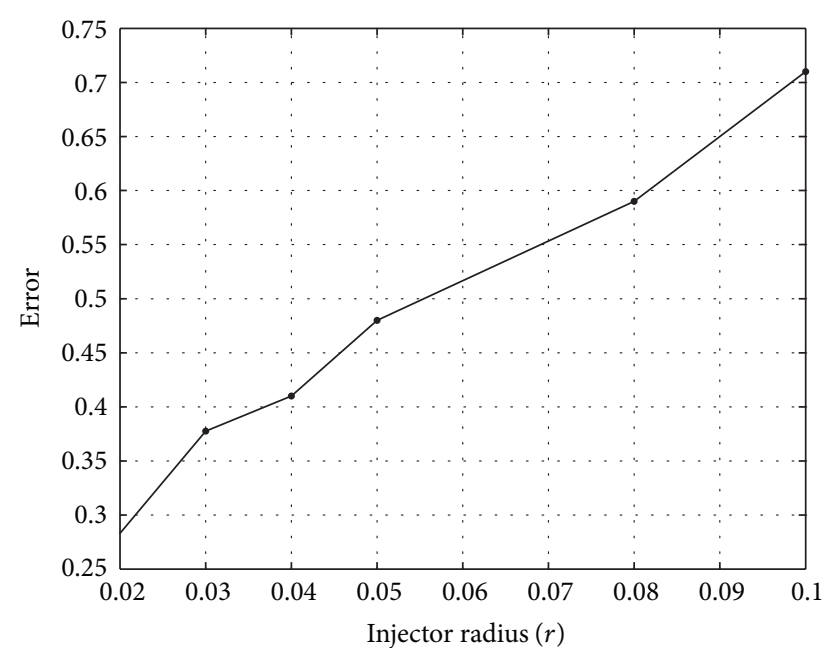

FIGURE 3: Injector radius effect.

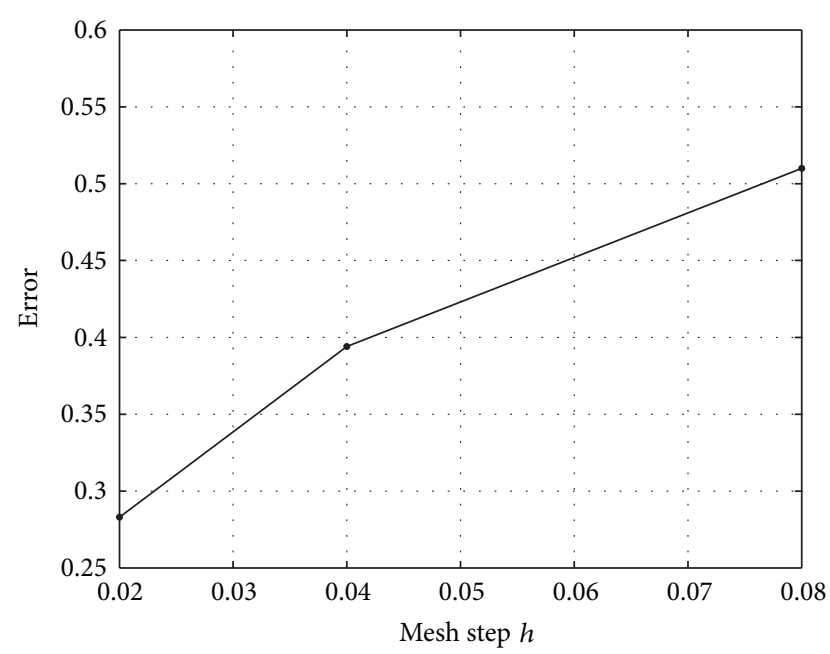

Figure 4: Mesh step effect.

4.2. Sensitivity to the Mesh Step. The mesh step effect is considered in this section. The error (35) is plotted in Figure 4 for a fixed radius $r=0.02$ and different mesh step $h$ varying from 0.02 to 0.08 . We remark that error rises when $h$ increases.

4.3. Detection of Separated Small Injectors. This test concerns the detection of optimal location of separated small injectors. Each injector is characterized by a small hole. In the numerical computation, we have considered the case of three injectors $\omega_{k}=z_{k}+r B(0,1), 1 \leq k \leq 3$, where $r<h$ and $h$ is the mesh size equal in this case to 0.02 .

The wanted velocity field $\mathcal{U}_{g}$ is chosen as solution to the Navier-Stokes problem in $\Omega \backslash \cup_{k=1}^{3} \overline{\omega_{z_{k}^{*}}}$, where $\omega_{z_{k}^{*}}=z_{k}^{*}+$ $h B(0,1), 1 \leq k \leq 3$ are the injectors to be detected. The exact locations $z_{k}^{*}$ and the injection velocities $u_{\text {inj }}^{k}$ are described in Table 1.

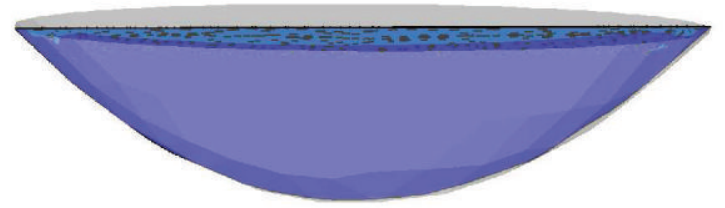

FIgURE 5: The initial flow.

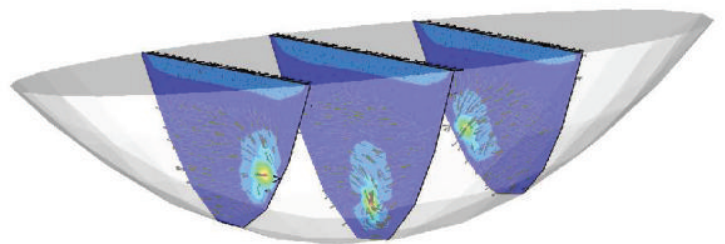

Figure 6: The wanted velocity: a 2D cut on each injector.

In Figure 5, we present the initial flow. The wanted flow is shown in Figure 6.

The isovalues of the topological sensitivity are plotted in Figure 7. Using Algorithm 4, the injectors locations are given by the local minima of the topological sensitivity $\delta j$. We show in Figure 7, respectively, on the left a vertical cut and on the right a horizontal cut of the topological sensitivity function $\delta j$ at each injector. One can observe that the local minima of the obtained topological sensitivity coincides with the exact location described in Table 1.

The obtained optimal locations are shown in Figure 8. The obtained velocity is shown in Figure 9. Since we have detected the exact location, the obtained velocity is identical to the wanted one.

4.4. Identification of an Injector Line. The aim here is the detection of an injector line $\sigma$ (see Figure 10). It is approximated by a sequence of small injectors (holes).

In order to detect $\sigma$, we propose here an iterative process based on the following algorithm.

Algorithm 5. (i) Initialization: choose $\Omega_{0}=\Omega$, and set $k=0$. (ii) Repeat until the target is reached:

(a) compute $u_{k}^{n}$ and $v_{k}^{n}$ for $n=1$, NT solutions, respectively, of (15) and (20);

(b) compute the topological sensitivity $\delta j_{k}$

$$
\begin{aligned}
\delta j_{k}=\sum_{n=1}^{\mathrm{NT}}[ & \left(u_{k}^{n}-u_{\mathrm{inj}}\right) \cdot v_{k}{ }^{n} \\
& \left.+\left(u_{k}^{n-1}-u_{\mathrm{inj}}\right) \cdot v_{k}^{n-1}\right], \quad \forall z \in \Omega,
\end{aligned}
$$

(c) determine the set $H_{k}=\left\{z \in \Omega_{k}, \delta j_{k}(z) \leq c_{k}<0\right\}$, where $c_{k}$ is chosen in such a way that the cost function decreases, 


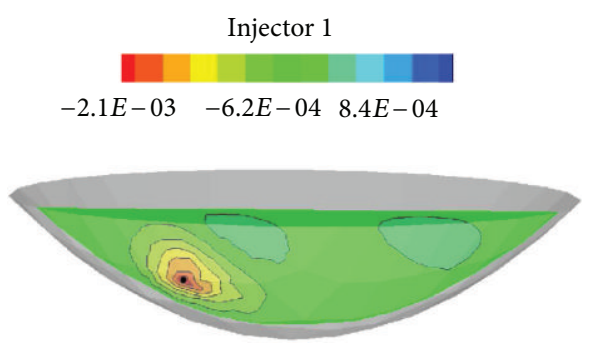

$2 \mathrm{D}$ cut on $y=-0.765$

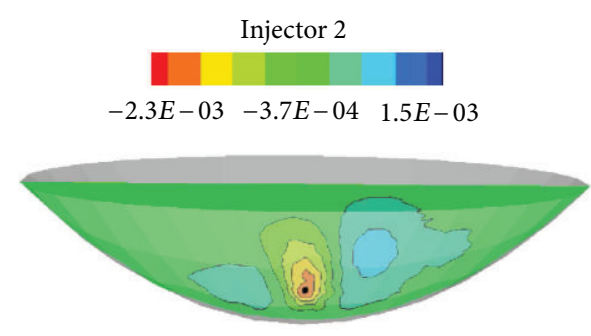

2D cut on $y=-0.058$

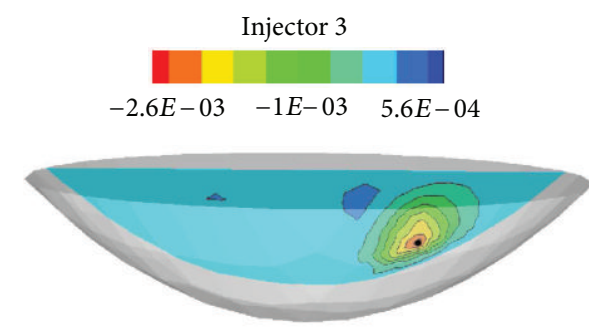

2D cut on $y=0.8$

(a)

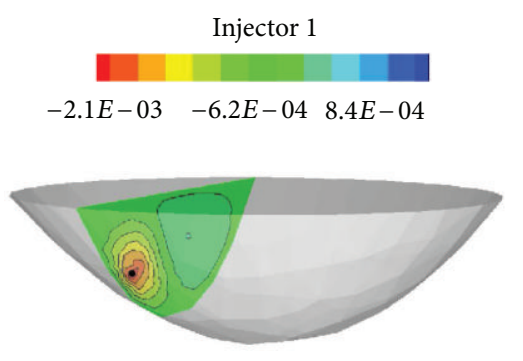

2D cut on $x=-1.599$

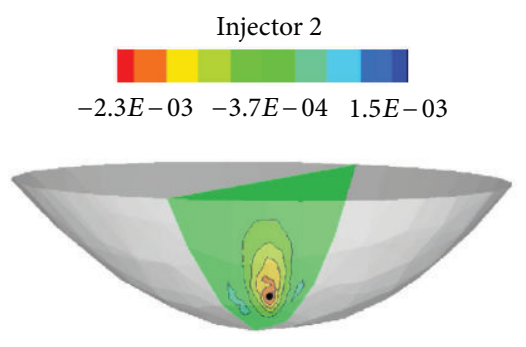

2D cut on $x=-0.035$

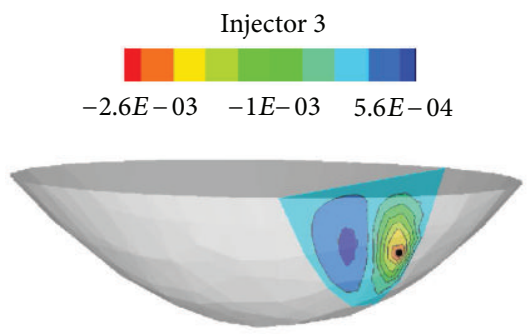

2D cut on $x=1.596$

(b)

FIGURE 7: 2D cuts of the topological sensitivity $\delta j$ showing the exact location (black dots) and the local minima of $\delta j$ (red zones).

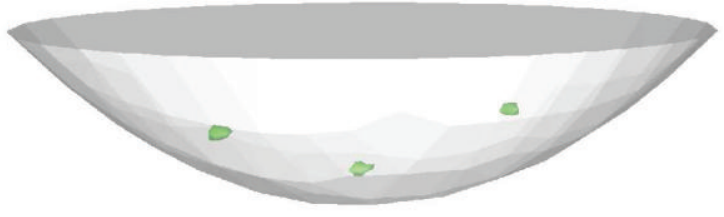

FIGURE 8: Obtained injectors location.

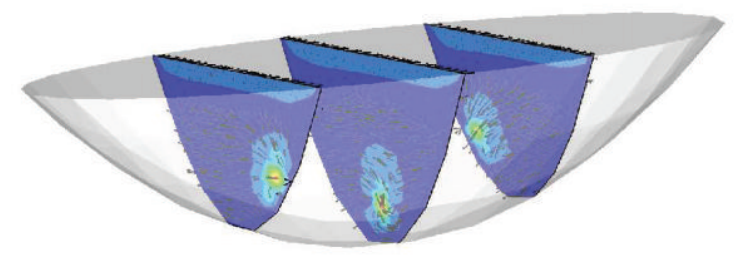

FIGURE 9: The obtained velocity: a 2D cut on each injector.

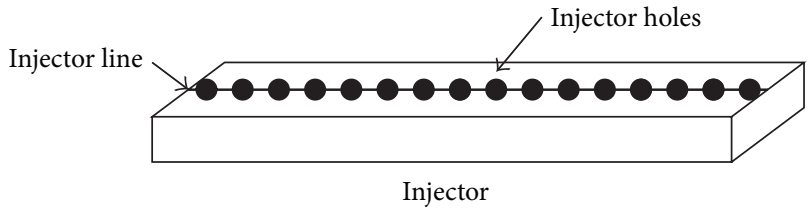

FIgURE 10: Injector.

(d) prescribe the velocity at each mesh node of $H_{k}: u(z)=$ $u_{\text {inj, }}$, for all $z \in H_{k}$,

(e) set $\Omega_{k+1}=\Omega_{k} \backslash H_{k}$,

(f) $k \leftarrow k+1$.

The injector $\sigma$ is detected iteratively, $\sigma_{k+1}=\sigma_{k} \cup H_{k}$, with $\sigma_{0}=\emptyset$. The constant $c_{k}$ depends on the most negative value of $\delta j_{k}$. In the presented test, $c_{k}=0.8 \delta_{k}$ with $\delta_{k}=\min _{z \in \Omega_{k}} \delta j_{k}(z)$.

The obtained results are shown in Figures 11-14. Figure 11(b) illustrates the obtained fluid flow created by the 
TABLE 1: The exact locations $z_{k}^{*}, 1 \leq k \leq 3$ and their associated injection velocities.

\begin{tabular}{lcc}
\hline Injector Inj $_{k}$ & \multicolumn{1}{c}{ Location $z_{k}^{*}$} & Injection velocity $u_{\text {inj }}^{k}$ \\
\hline Inj $_{1}$ & $x=-1.599, y=-0.765, z=-1.041$ & $u_{x}=-0.3, u_{y}=0.0, u_{z}=-0.4$ \\
Inj $_{2}$ & $x=-0.035, y=-0.058, z=-1.565$ & $u_{x}=0.0, u_{y}=0.0, u_{z}=0.5$ \\
Inj $_{3}$ & $x=1.0, y=0.800, z=-1.023$ & $u_{x}=-0.2, u_{y}=0.0, u_{z}=0.4$ \\
\hline
\end{tabular}

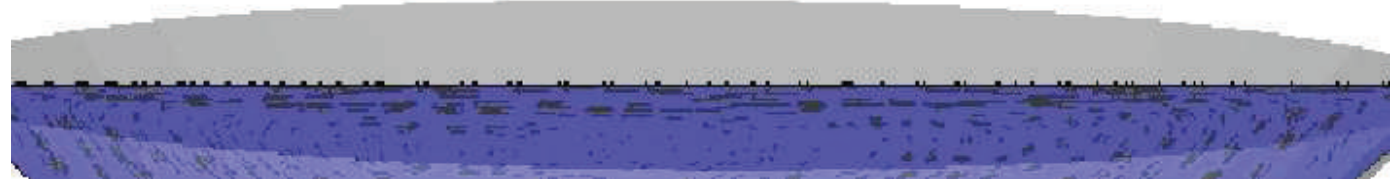

(a) $u_{g}$

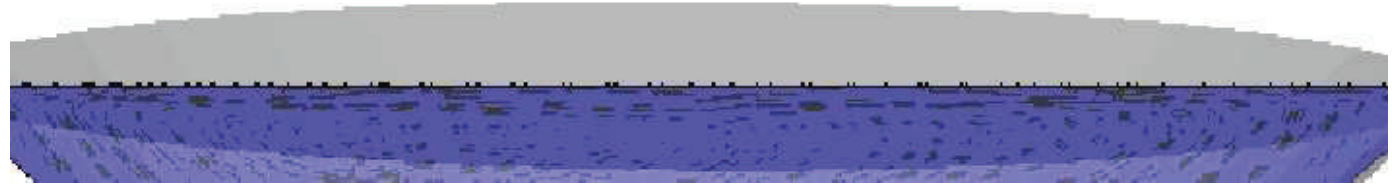

(b) $u_{\varepsilon}$ after 3 iterations.

FIGURE 11: The wanted and obtained fluid flow on the measurement domain $\Omega_{m}$.

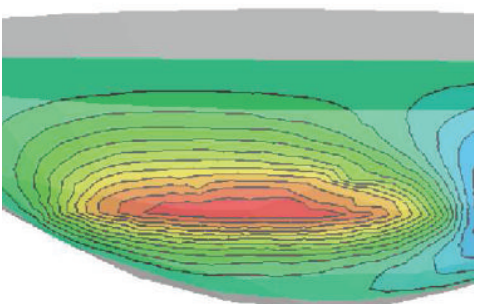

First iteration

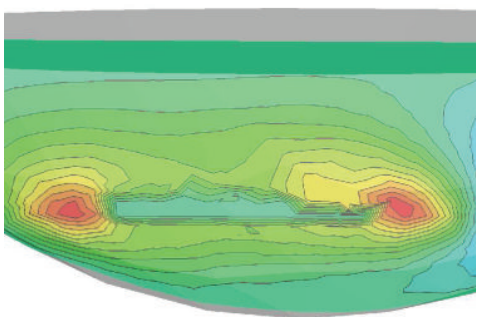

Second iteration

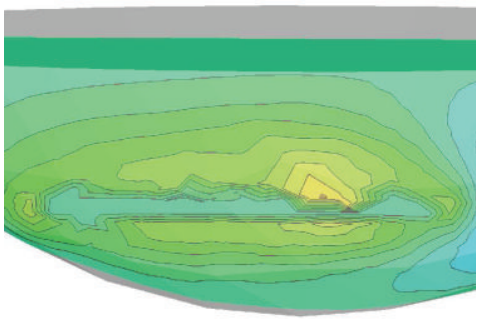

Third iteration

(a)

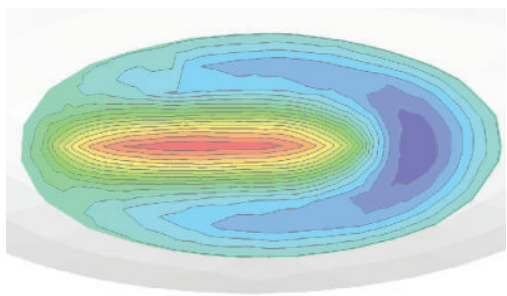

First iteration

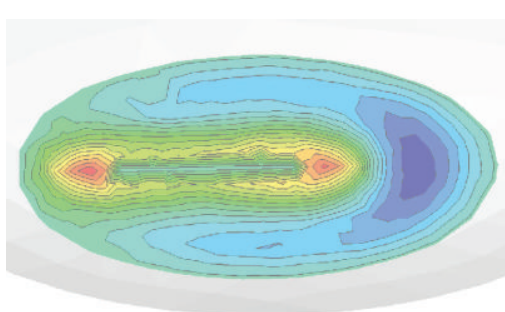

Second iteration

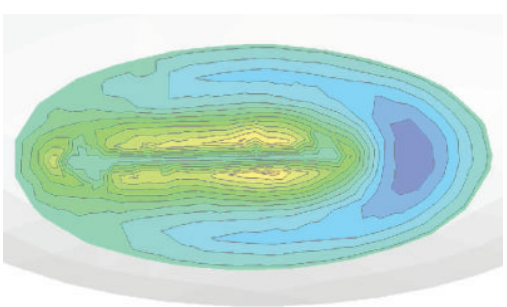

Third iteration

(b)

FIGURE 12: Isovalues of $\delta j_{k}$ showing the detected zone $H_{k}$ (red zone) during the optimization process: a vertical cut (a), a horizontal cut (b). 


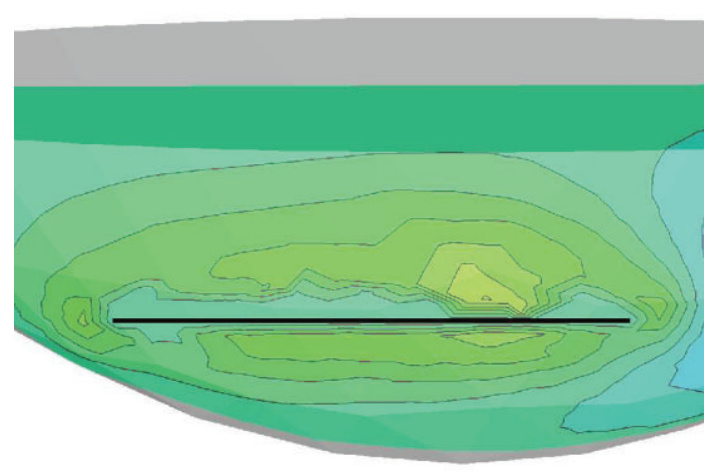

FIGURE 13: Injector position with respect to $\delta j_{3}$.

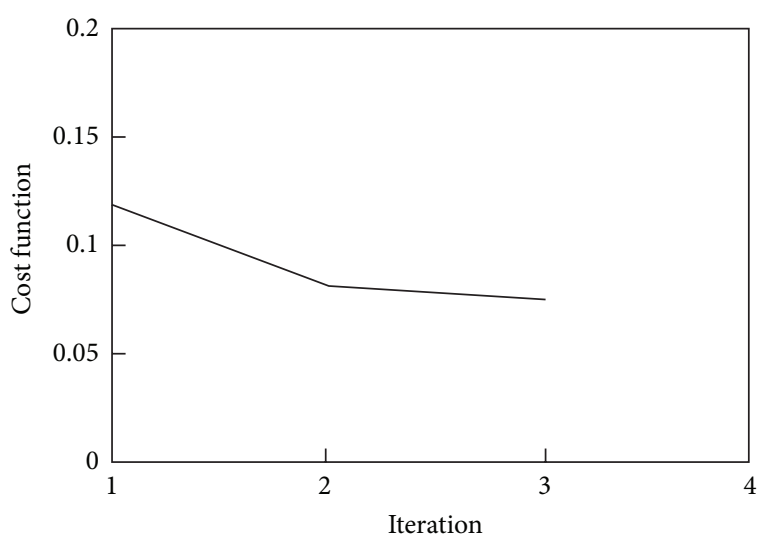

FIGURE 14: Objective function $\mathscr{J}_{\varepsilon}\left(u_{\varepsilon}\right)$.

detected injector. It is nearly identical to the wanted one (see Figure 11(a)).

In Figure 12 we plot the isovalues of $\delta j_{k}$ showing the detected zone $H_{k}$ (where $\delta j_{k}$ is most negative) during the optimization process.

One can note that the proposed algorithm (Algorithm 5) permits to detect the injector line in only three iterations (Figure 13). Finally Figure 14 plots the objective function $\mathscr{J}_{\varepsilon}\left(u_{\varepsilon}\right)$.

\section{Conclusion}

In this paper a numerical study of the optimal injector location problem in lake aeration process has been studied. The topological sensitivity analysis method is used to solve the optimization problem. The proposed numerical algorithm is fast, is easily implemented, and only needs to solve the direct and the adjoint problems on a fixed grid. In practice we have obtained interesting numerical results. Like all numerical methods, the proposed approach has its own drawbacks: on one hand, in the case of the simultaneous creation of multiple holes. The topological sensitivity $\delta j$ provides information on where to create holes independently of its number. Then the obtained solution can be seen rather as a suggestion then as an optimal solution. On the other hand, the numerical realization via discretization may not take properly into account the correct influence of the shape of the hole assumed to be spherical of radius $\varepsilon \rightarrow 0$. To this aim we need a very fine grid which leads to many difficulties mainly due to the high computing costs necessary for such three-dimensional application. For this reason a parallel implementation of the numerical algorithm can be investigated in a forthcoming work.

\section{Acknowledgments}

This project was supported by King Saud University, Deanship of Scientific Research, College of Science Research Center.

\section{References}

[1] M. Hassine, S. Jan, and M. Masmoudi, "From differential calculus to 0-1 topological optimization," SIAM Journal on Control and Optimization, vol. 45, no. 6, pp. 1965-1987, 2007.

[2] M. Masmoudi, "The topological asymptotic," in Computational Methods for Control Applications, H. Kawarada and J. Periaux, Eds., International Series GAKUTO, 2002.

[3] J. Sokołowski and A. Zochowski, "On the topological derivative in shape optimization," SIAM Journal on Control and Optimization, vol. 37, no. 4, pp. 1251-1272, 1999.

[4] M. Abdelwahed, M. Hassine, and M. Masmoudi, "Control of a mechanical aeration process via topological sensitivity analysis," Journal of Computational and Applied Mathematics, vol. 228, no. 1, pp. 480-485, 2009.

[5] M. Ishii, Thermo-fluid dynamic theory of a two-phase flow, Collection de la direction des études de recherche d'électricité de france, Librairie Eyrolles, Paris, France, 1975.

[6] D. Legendre, Quelques aspects des forces hydrodynamiques et des transferts de chaleur sur une bulle spherique [Ph.D. thesis], INP Toulouse, Toulouse, France, 1996.

[7] E. Clement, Dispertion de bulles et modifications du mouvement de la phase porteuse dans des ecoulements tourbillonnaires [Ph.D. thesis], Institut Nationale polytechnique de Toulouse, Toulouse, France, 1999.

[8] M. Abdelwahed, F. Dabaghi, and D. Ouazar, "A virtual numerical simulator for aeration effects in lake eutrophication," International Journal of Computational Fluid Dynamics, vol. 16, no. 2, pp. 119-128, 2002.

[9] S. Amstutz, "The topological asymptotic for the Navier-Stokes equations," ESAIM. Control, Optimisation and Calculus of Variations, vol. 11, no. 3, pp. 401-425, 2005.

[10] Ph. Guillaume and K. Sid Idris, "Topological sensitivity and shape optimization for the Stokes equations," SIAM Journal on Control and Optimization, vol. 43, no. 1, pp. 1-31, 2004.

[11] M. Hassine and M. Masmoudi, "The topological asymptotic expansion for the quasi-Stokes problem," ESAIM, Control, Optimization and Calculus of Variations, vol. 10, no. 4, pp. 478504, 2004.

[12] E. Suli, "Convergence and nonlinear stability of the LagrangeGalerkin method for the Navier-Stokes equations," Numerische Mathematik, vol. 53, no. 4, pp. 459-483, 1988.

[13] O. Pironneau, Méthode des Éléments Finis Pour les fluides, Masson, Paris, France, 1998. 
[14] M. Abdelwahed, Modélisation et simulation numérique d'écoulements diphasiques [Ph.D. thesis], Pau University, Pau, France, 2002.

[15] M. Abdelwahed, M. Hassine, and M. Masmoudi, "Optimal shape design for fluid flow using topological perturbation technique," Journal of Mathematical Analysis and Applications, vol. 356, no. 2, pp. 548-563, 2009.

[16] D. N. Arnold, F. Brezzi, and M. Fortin, "A stable finite element for the Stokes equations," Calcolo, vol. 21, no. 4, pp. 337-344, 1984. 


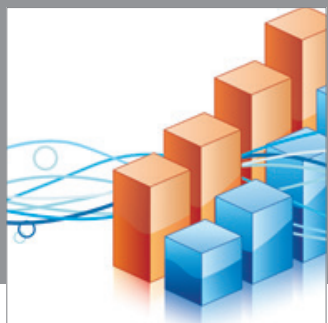

Advances in

Operations Research

mansans

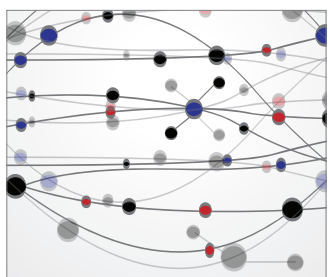

The Scientific World Journal
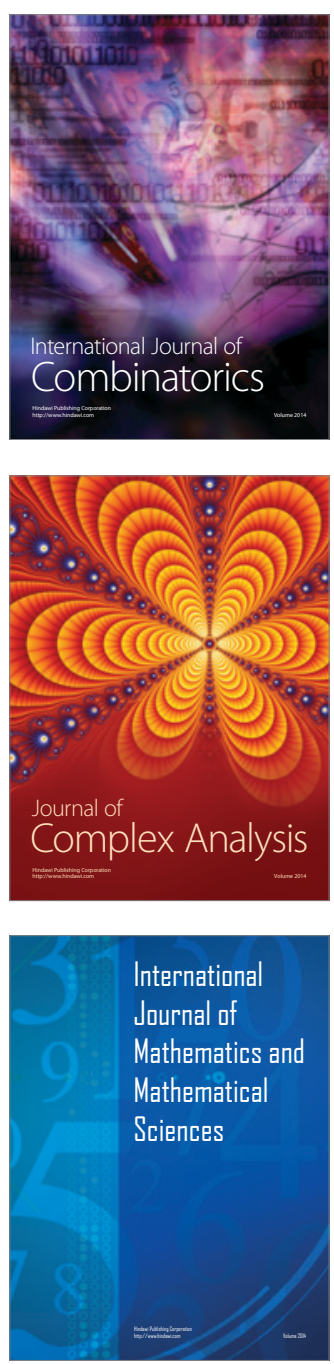
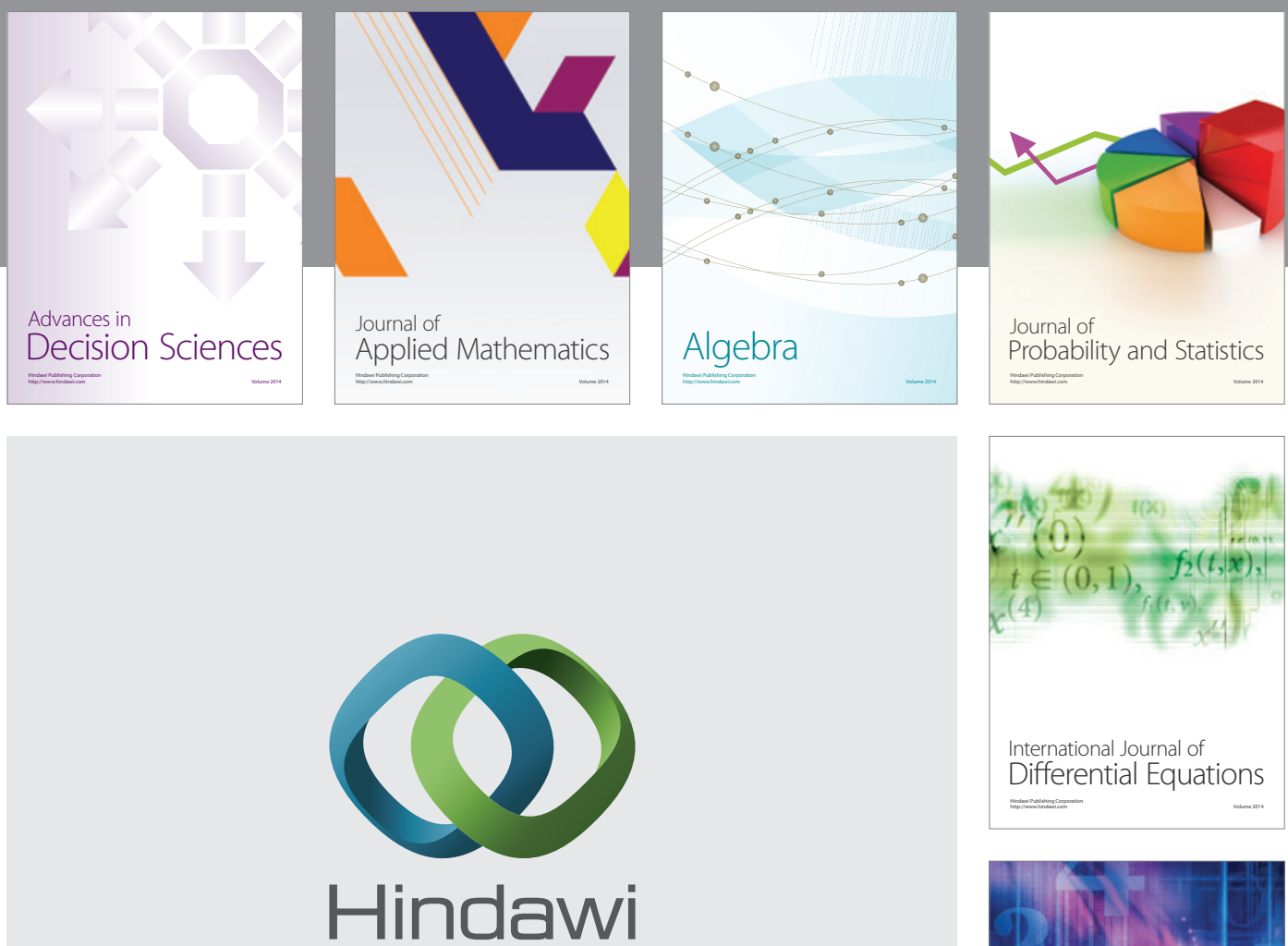

Submit your manuscripts at http://www.hindawi.com
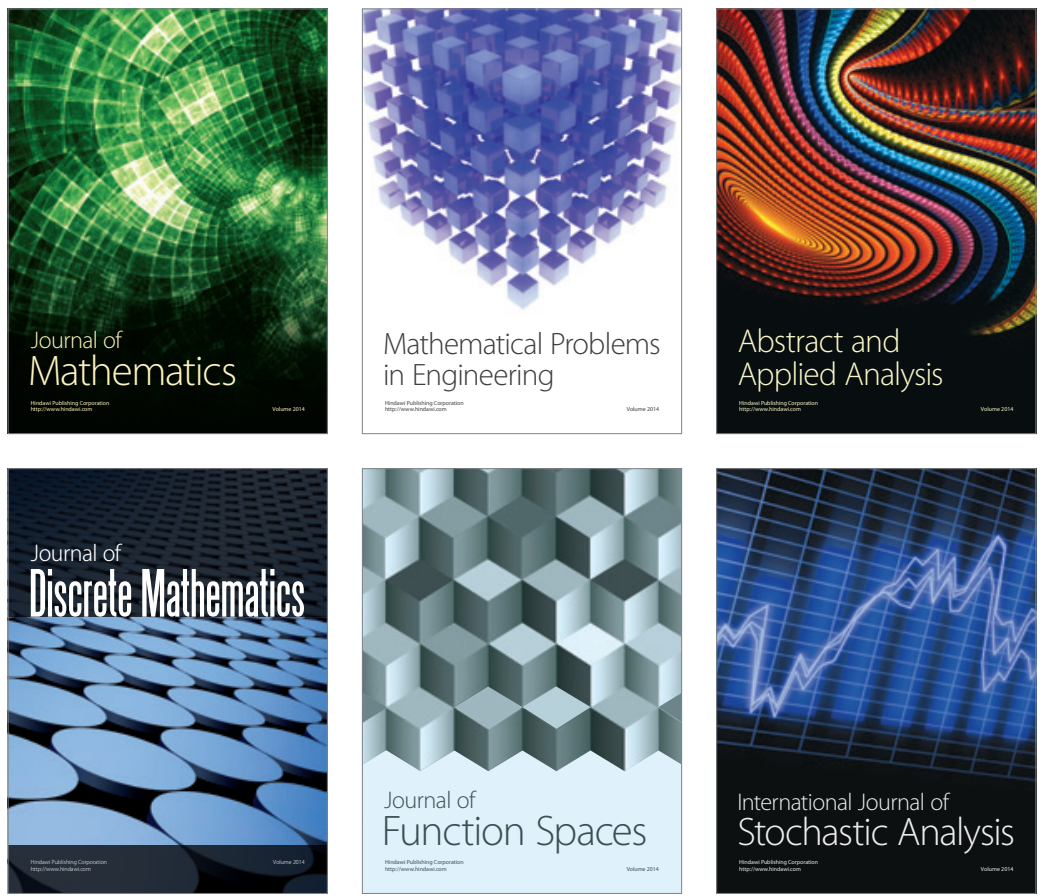

Journal of

Function Spaces

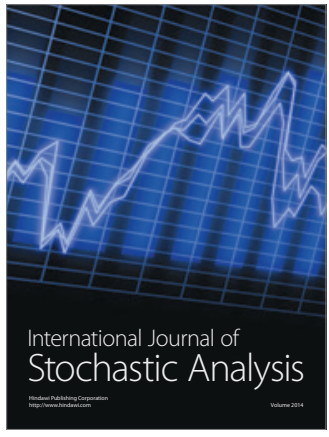

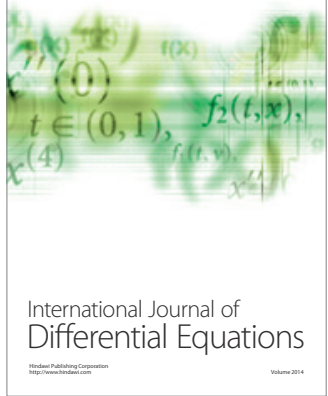
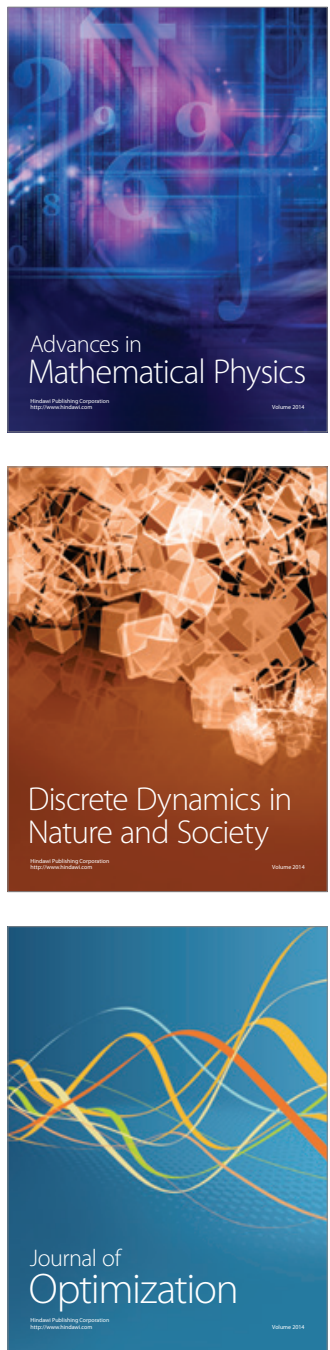